\title{
Nonexistence of Homoclinic Solutions for a Class of Discrete Hamiltonian Systems
}

\author{
Xiaoping Wang \\ Department of Mathematics, Xiangnan College, Chenzhou, Hunan 423000, China \\ Correspondence should be addressed to Xiaoping Wang; wxp31415@sina.com
}

Received 3 September 2012; Accepted 27 November 2012

Academic Editor: Jinde Cao

Copyright (C) 2013 Xiaoping Wang. This is an open access article distributed under the Creative Commons Attribution License, which permits unrestricted use, distribution, and reproduction in any medium, provided the original work is properly cited.

We give several sufficient conditions under which the first-order nonlinear discrete Hamiltonian system $\Delta x(n)=\alpha(n) x(n+$ $1)+\beta(n)|y(n)|^{\mu-2} y(n), \Delta y(n)=-\gamma(n)|x(n+1)|^{\nu-2} x(n+1)-\alpha(n) y(n)$ has no solution $(x(n), y(n))$ satisfying condition $0<$ $\sum_{n=-\infty}^{+\infty}\left[|x(n)|^{\nu}+(1+\beta(n))|y(n)|^{\mu}\right]<+\infty$, where $\mu, v>1$ and $1 / \mu+1 / \nu=1$ and $\alpha(n), \beta(n)$, and $\gamma(n)$ are real-valued functions defined on $\mathbb{Z}$.

\section{Introduction}

In 1907, Lyapunov [1] established the first so-called Lyapunov inequality:

$$
(b-a) \int_{a}^{b} q(t) d t>4
$$

if Hill's equation

$$
x^{\prime \prime}(t)+q(t) x(t)=0
$$

has a real solution $x(t)$ such that

$$
x(a)=x(b)=0, \quad x(t) \not \equiv 0, \quad t \in[a, b],
$$

and the constant 4 in (1) cannot be replaced by a larger number, where $q(t)$ is a piecewise continuous and nonnegative function defined on $\mathbb{R}$. Since this result has found applications in the study of various properties of solutions such as oscillation theory, disconjugacy, and eigenvalue problems of (2), a large number of Lyapunov-type inequalities were established in the literature which generalized or improved (1); see [1-20].

In 1983, Cheng [3] first obtained the discrete analogy of Lyapunov inequality (1) for the second-order difference equation:

$$
\Delta^{2} x(n)+q(n) x(n+1)=0
$$

where, and in the sequel, $\Delta$ denotes the forward difference operator defined by $\Delta x(n)=x(n+1)-x(n)$.

When $a=-\infty$ and $b=+\infty$, that is, system (4) has a solution $x(n)$ satisfying $\lim _{|n| \rightarrow \infty} x(n)=0$, which is called homoclinic solution, whether one can obtain Lyapunov-type inequalities for (4)? To the best of our knowledge, there are no results.

In 2003, Sh. Guseinov and Kaymakçalan [7] partly generalized the Cheng's result to the discrete linear Hamiltonian system:

$$
\begin{gathered}
\Delta x(n)=\alpha(n) x(n+1)+\beta(n) y(n), \\
\Delta y(n)=-\gamma(n) x(n+1)-\alpha(n) y(n),
\end{gathered}
$$

where $\alpha(n), \beta(n)$, and $\gamma(n)$ are real-valued functions defined on $\mathbb{Z}$ and $a$ and $b$ are not necessarily usual zeros, but rather, generalized zeros. Later, some better Lyapunov-type inequalities for system (5) were obtained in $[19,20]$.

Very recently, He and Zhang [10] further generalized the result in [19] to the following first-order nonlinear difference system:

$$
\begin{gathered}
\Delta x(n)=\alpha(n) x(n+1)+\beta(n)|y(n)|^{\mu-2} y(n), \\
\Delta y(n)=-\gamma(n)|x(n+1)|^{\nu-2} x(n+1)-\alpha(n) y(n),
\end{gathered}
$$

where $\mu, \nu>1$ and $1 / \mu+1 / \nu=1$ and $\alpha(n), \beta(n)$, and $\gamma(n)$ are real-valued functions defined on $\mathbb{Z}$. 
When $\mu=\nu=2$, system (6) reduces to (5). In addition, the special forms of system (6) contain many well-known difference equations which have been studied extensively and have much applications in the literature [21-23], such as the second-order linear difference equation:

$$
\Delta[p(n) \Delta x(n)]+q(n) x(n+1)=0,
$$

and the second-order half-linear difference equation:

$$
\Delta\left[p(n)|\Delta x(n)|^{r-2} \Delta x(n)\right]+q(n)|x(n+1)|^{r-2} x(n+1)=0,
$$

where $r>1, p(n)$ and $q(n)$ are real-valued functions defined on $\mathbb{Z}$ and $p(n)>0$. Let

$$
y(n)=p(n)|\Delta x(n)|^{r-2} \Delta x(n),
$$

then (8) can be written as the form of (6):

$$
\begin{gathered}
\Delta x(n)=[p(n)]^{1 /(1-r)}|y(n)|^{(2-r) /(r-1)} y(n), \\
\Delta y(n)=-q(n)|x(n+1)|^{r-2} x(n+1),
\end{gathered}
$$

where $\mu=r /(r-1), v=r$ and $\alpha(n)=0, \beta(n)=[p(n)]^{1 /(1-r)}$ and $\gamma(n)=q(n)$.

In this paper, we will establish several Lyapunov-type inequalities for systems (5) and (6) if they have a solution $(x(n), y(n))$ satisfying conditions

$$
\begin{aligned}
& 0<\sum_{-\infty}^{+\infty}\left[|x(n)|^{2}+(1+\beta(n))|y(n)|^{2}\right]<+\infty, \\
& 0<\sum_{-\infty}^{+\infty}\left[|x(n)|^{\nu}+(1+\beta(n))|y(n)|^{\mu}\right]<+\infty,
\end{aligned}
$$

respectively. Taking advantage of these Lyapunov-type inequalities, we are able to establish some criteria for nonexistence of homoclinic solutions of systems (5) and (6). As we know, there are no results on non-existence of homoclinic solutions for Hamiltonian systems in previous literature.

\section{Lyapunov-Type Inequalities for System (6)}

In this section, we shall establish some Lyapunov-type inequalities for system (6). For the sake of convenience, we list some assumptions on $\alpha(n)$ and $\beta(n)$ as follows:

(A0) $\alpha(n)<1$, for all $n \in \mathbb{Z}, \prod_{s=-\infty}^{+\infty}[1-\alpha(s)]^{-1}<\infty$;

(A1) $\alpha(n)<1$, for all $n \in \mathbb{Z}, \sum_{s=-\infty}^{+\infty}|\alpha(s)|<+\infty$;

(B0) $\beta(n) \geq($ (三) 0 , for all $n \in \mathbb{Z}$;

(B1) $\sum_{\tau=-\infty}^{0} \beta(\tau) \prod_{s=\tau}^{0}[1-\alpha(s)]^{-\mu}$ $+\sum_{\tau=1}^{+\infty} \beta(\tau) \prod_{s=0}^{\tau-1}[1-\alpha(s)]^{\mu}<+\infty$.

Denote

$$
\begin{aligned}
& \zeta(n):=\left[\sum_{\tau=-\infty}^{n} \beta(\tau) \prod_{s=\tau}^{n}[1-\alpha(s)]^{-\mu}\right]^{\nu / \mu}, \\
& \eta(n):=\left[\sum_{\tau=n+1}^{+\infty} \beta(\tau) \prod_{s=n+1}^{\tau-1}[1-\alpha(s)]^{\mu}\right]^{\nu / \mu} .
\end{aligned}
$$

Theorem 1. Suppose that hypotheses (A0), (B0), and (B1) are satisfied. If system (6) has a solution $(x(n), y(n))$ satisfying

$$
0<\sum_{n=-\infty}^{+\infty}\left[|x(n)|^{\nu}+(1+\beta(n))|y(n)|^{\mu}\right]<+\infty
$$

then one has the following inequality:

$$
\sum_{n=-\infty}^{+\infty} \frac{\zeta(n) \eta(n)}{\zeta(n)+\eta(n)} \gamma^{+}(n) \geq 1
$$

where $\gamma^{+}(n)=\max \{\gamma(n), 0\}$.

Proof. Hypothesis (B1) implies that functions $\zeta(n)$ and $\eta(n)$ are well defined on $\mathbb{Z}$. Without loss of generality, we can assume that

$$
\sum_{n=-\infty}^{+\infty} \frac{\zeta(n) \eta(n)}{\zeta(n)+\eta(n)} \gamma^{+}(n)<+\infty
$$

From (14) and (B0), one has

$$
\begin{gathered}
\lim _{|n| \rightarrow \infty}|x(n)|=\lim _{|n| \rightarrow \infty}|y(n)|=0, \\
\sum_{\tau=-\infty}^{+\infty} \beta(\tau)|y(\tau)|^{\mu}<+\infty .
\end{gathered}
$$

It follows from (13), (18), and the Hölder inequality that

$$
\begin{aligned}
& \sum_{\tau=-\infty}^{n} \beta(\tau)|y(\tau)|^{\mu-1} \prod_{s=\tau}^{n}[1-\alpha(s)]^{-1} \\
& \quad \leq\left[\sum_{\tau=-\infty}^{n} \beta(\tau) \prod_{s=\tau}^{n}[1-\alpha(s)]^{-\mu}\right]^{1 / \mu}\left[\sum_{\tau=-\infty}^{n} \beta(\tau)|y(\tau)|^{\mu}\right]^{1 / v} \\
& \quad=[\zeta(n)]^{1 / v}\left[\sum_{\tau=-\infty}^{n} \beta(\tau)|y(\tau)|^{\mu}\right]^{1 / v} \\
& \quad<+\infty, \quad \forall n \in \mathbb{Z},
\end{aligned}
$$

$$
\begin{aligned}
& \sum_{\tau=n+1}^{+\infty} \beta(\tau)|y(\tau)|^{\mu-1} \prod_{s=n+1}^{\tau-1}[1-\alpha(s)] \\
& \leq\left[\sum_{\tau=n+1}^{+\infty} \beta(\tau) \prod_{s=n+1}^{\tau-1}[1-\alpha(s)]^{\mu}\right]^{1 / \mu}\left[\sum_{\tau=n+1}^{+\infty} \beta(\tau)|y(\tau)|^{\mu}\right]^{1 / v} \\
& \quad=[\eta(n)]^{1 / v}\left[\sum_{\tau=n+1}^{+\infty} \beta(\tau)|y(\tau)|^{\mu}\right]^{1 / v} \\
& \quad<+\infty, \quad \forall n \in \mathbb{Z} .
\end{aligned}
$$


From (A0), (17), (19), (20), and the first equation of system (6), we have

$$
\begin{array}{ll}
x(n+1)=\sum_{\tau=-\infty}^{n} \beta(\tau)|y(\tau)|^{\mu-2} y(\tau) \prod_{s=\tau}^{n}[1-\alpha(s)]^{-1}, & \forall n \in \mathbb{Z}, \\
x(n+1)=-\sum_{\tau=n+1}^{+\infty} \beta(\tau)|y(\tau)|^{\mu-2} y(\tau) \prod_{s=n+1}^{\tau-1}[1-\alpha(s)], & \forall n \in \mathbb{Z} .
\end{array}
$$

Combining (19) with (21), one has

$$
\begin{aligned}
|x(n+1)|^{\nu} & =\left.\left.\left|\sum_{\tau=-\infty}^{n} \beta(\tau)\right| y(\tau)\right|^{\mu-2} y(\tau) \prod_{s=\tau}^{n}[1-\alpha(s)]^{-1}\right|^{\nu} \\
& \leq \zeta(n) \sum_{\tau=-\infty}^{n} \beta(\tau)|y(\tau)|^{\mu}, \quad \forall n \in \mathbb{Z} .
\end{aligned}
$$

Similarly, it follows from (20) and (22) that

$$
\begin{aligned}
|x(n+1)|^{\nu} & =\left.\left.\left|\sum_{\tau=n+1}^{+\infty} \beta(\tau)\right| y(\tau)\right|^{\mu-2} y(\tau) \prod_{s=n+1}^{\tau-1}[1-\alpha(s)]\right|^{\nu} \\
& \leq \eta(n) \sum_{\tau=n+1}^{+\infty} \beta(\tau)|y(\tau)|^{\mu}, \quad \forall n \in \mathbb{Z} .
\end{aligned}
$$

Combining (23) with (24), one has

$$
|x(n+1)|^{\nu} \leq \frac{\zeta(n) \eta(n)}{\zeta(n)+\eta(n)} \sum_{\tau=-\infty}^{+\infty} \beta(\tau)|y(\tau)|^{\mu}, \quad \forall n \in \mathbb{Z} .
$$

Now, it follows from (16), (18), and (25) that

$$
\begin{aligned}
\sum_{n=-\infty}^{+\infty} \gamma^{+}(n)|x(n+1)|^{\nu} & \\
\leq & {\left[\sum_{n=-\infty}^{+\infty} \frac{\zeta(n) \eta(n)}{\zeta(n)+\eta(n)} \gamma^{+}(n)\right] } \\
& \times \sum_{n=-\infty}^{+\infty} \beta(n)|y(n)|^{\mu}<+\infty .
\end{aligned}
$$

By (6), we obtain

$$
\Delta(x(n) y(n))=\beta(n)|y(n)|^{\mu}-\gamma(n)|x(n+1)|^{\nu} .
$$

Summing the above from $-\infty$ to $+\infty$ and using (17) and (18), we obtain

$$
\sum_{n=-\infty}^{+\infty} \gamma(n)|x(n+1)|^{\nu}=\sum_{n=-\infty}^{+\infty} \beta(n)|y(n)|^{\mu}
$$

which, together with (26), implies that

$$
\begin{aligned}
& \sum_{n=-\infty}^{+\infty} \gamma^{+}(n)|x(n+1)|^{\nu} \\
& \leq\left[\sum_{n=-\infty}^{+\infty} \frac{\zeta(n) \eta(n)}{\zeta(n)+\eta(n)} \gamma^{+}(n)\right] \sum_{n=-\infty}^{+\infty} \beta(n)|y(n)|^{\mu} \\
& \quad=\left[\sum_{n=-\infty}^{+\infty} \frac{\zeta(n) \eta(n)}{\zeta(n)+\eta(n)} \gamma^{+}(n)\right] \sum_{n=-\infty}^{+\infty} \gamma(n)|x(n+1)|^{\nu} \\
& \leq\left[\sum_{n=-\infty}^{+\infty} \frac{\zeta(n) \eta(n)}{\zeta(n)+\eta(n)} \gamma^{+}(n)\right] \sum_{n=-\infty}^{+\infty} \gamma^{+}(n)|x(n+1)|^{\nu} .
\end{aligned}
$$

We claim that

$$
\sum_{n=-\infty}^{+\infty} \gamma^{+}(n)|x(n+1)|^{\nu}>0
$$

If (30) is not true, then

$$
\sum_{n=-\infty}^{+\infty} \gamma^{+}(n)|x(n+1)|^{\nu}=0
$$

From (28) and (31), we have

$$
\begin{aligned}
0 & \leq \sum_{n=-\infty}^{+\infty} \beta(n)|y(n)|^{\mu}=\sum_{n=-\infty}^{+\infty} \gamma(n)|x(n+1)|^{\nu} \\
& \leq \sum_{n=-\infty}^{+\infty} \gamma^{+}(n)|x(n+1)|^{\nu}=0 .
\end{aligned}
$$

It follows that

$$
\beta(n)|y(n)|^{\mu-2} y(n) \equiv 0, \quad \forall n \in \mathbb{Z} .
$$

Combining (21) with (33), we obtain that

$$
x(n) \equiv 0, \quad \forall n \in \mathbb{Z},
$$

which, together with the second equation of system (6), implies that

$$
\Delta y(n)=-\alpha(n) y(n), \quad \forall n \in \mathbb{Z} .
$$

Combining the above with (17), one has

$$
y(n) \equiv 0, \quad \forall n \in \mathbb{Z} .
$$

Both (34) and (36) contradict with (14). Therefore, (30) holds. Hence, it follows from (29) and (30) that (15) holds.

Corollary 2. Suppose that hypotheses (A1), (B0), and (B1) are satisfied. If system (6) has a solution $(x(n), y(n))$ satisfying (14), then one has the following inequality:

$$
\sum_{n=-\infty}^{+\infty} \gamma^{+}(n)\left[\sum_{\tau=-\infty}^{n} \beta(\tau) \sum_{\tau=n+1}^{+\infty} \beta(\tau)\right]^{\nu / 2 \mu} \geq 2 \prod_{n=-\infty}^{+\infty}\{\Theta[\alpha(n)]\}^{\nu / 2},
$$


where and in the sequel,

$$
\begin{gathered}
\Theta[\alpha(n)]=\min \left\{1-\alpha^{+}(n),\left[1+\alpha^{-}(n)\right]^{-1}\right\}, \\
\alpha^{+}(n)=\max \{\alpha(n), 0\}, \quad \alpha^{-}(n)=\max \{-\alpha(n), 0\} .
\end{gathered}
$$

Proof. Obviously, (A1) implies that

$$
0<\prod_{s=-\infty}^{+\infty}[1-\alpha(s)]<+\infty,
$$

and so (A0) holds, and which, together with (B1), implies that $\sum_{\tau=-\infty}^{+\infty} \beta(\tau)<+\infty$. Since

$$
\zeta(n)+\eta(n) \geq 2[\zeta(n) \eta(n)]^{1 / 2},
$$

it follows that

$$
\begin{aligned}
1 \leq & \sum_{n=-\infty}^{+\infty} \frac{\zeta(n) \eta(n)}{\zeta(n)+\eta(n)} \gamma^{+}(n) \\
\leq & \frac{1}{2} \sum_{n=-\infty}^{+\infty}[\zeta(n) \eta(n)]^{1 / 2} \gamma^{+}(n) \\
= & \frac{1}{2} \sum_{n=-\infty}^{+\infty} \gamma^{+}(n)\left\{\sum_{\tau=-\infty}^{n} \beta(\tau) \prod_{s=\tau}^{n}[1-\alpha(s)]^{-\mu}\right. \\
& \left.\times \sum_{\tau=n+1}^{+\infty} \beta(\tau) \prod_{s=n+1}^{\tau-1}[1-\alpha(s)]^{\mu}\right\}^{v / 2 \mu} \\
\leq & \sum_{n=-\infty}^{+\infty} \gamma^{+}(n)\left\{\sum_{\tau=-\infty}^{n} \beta(\tau) \prod_{s=\tau}^{n}\left[1-\alpha^{+}(s)\right]^{-\mu}\right. \\
\leq & \frac{1}{2} \sum_{n=-\infty}^{+\infty} \gamma^{+}(n)\left[\sum_{\tau=-\infty}^{n} \beta(\tau) \sum_{\tau=n+1}^{+\infty} \beta(\tau) \prod_{s=n+1}^{+\infty}\left[1+\alpha^{-}(s)\right]^{\mu}\right\} \\
& \times \prod_{s=-\infty}^{n}\left[1-\alpha^{+}(s)\right]^{-v / 2} \prod_{s=n+1}^{+\infty}\left[1+\alpha^{-}(s)\right]^{\nu / 2} \\
\leq & \frac{1}{2} \sum_{n=-\infty}^{+\infty} \gamma^{+}(n)\left[\sum_{\tau=-\infty}^{n} \beta(\tau) \sum_{\tau=n+1}^{b} \beta(\tau)\right]^{\nu / 2 \mu} \\
& \times \prod_{s=-\infty}^{+\infty}\{\Theta[\alpha(s)]\}^{-v / 2},
\end{aligned}
$$

which implies that (37) holds.

Since

$$
\left[\sum_{\tau=-\infty}^{n} \beta(\tau) \sum_{\tau=n+1}^{+\infty} \beta(\tau)\right]^{1 / 2} \leq \frac{1}{2} \sum_{n=-\infty}^{+\infty} \beta(n),
$$

then it follows from (37) that the following corollary is true.
Corollary 3. Suppose that hypotheses (A1), (B0), and (B1) are satisfied. If system (6) has a solution $(x(n), y(n))$ satisfying (14), then

$$
\left(\sum_{n=-\infty}^{+\infty} \beta(n)\right)^{1 / \mu}\left(\sum_{n=-\infty}^{+\infty} \gamma^{+}(n)\right)^{1 / v} \geq 2 \prod_{n=-\infty}^{+\infty}\{\Theta[\alpha(n)]\}^{1 / 2} .
$$

Applying Theorem 1 and Corollary 2 to system (8) (i.e., (10)), we have immediately the following two corollaries.

Corollary 4. Suppose that $r>1$ and $p(n)>0$ for $n \in \mathbb{Z}$, and that

$$
\sum_{\tau=-\infty}^{+\infty} \frac{1}{[p(\tau)]^{1 /(r-1)}}<+\infty
$$

If (8) has a solution $x(n)$ satisfying

$$
0<\sum_{n=-\infty}^{+\infty}\left[|x(n)|^{r}+p(n)\left(1+[p(n)]^{1 /(r-1)}\right)|\Delta x(n)|^{r}\right]<+\infty,
$$

then

$$
\begin{aligned}
\sum_{n=-\infty}^{+\infty}\left(\left\{\sum_{\tau=-\infty}^{n}[p(\tau)]^{-1 /(r-1)}\right\}^{r-1}\left\{\sum_{\tau=n+1}^{+\infty}[p(\tau)]^{-1 /(r-1)}\right\}^{r-1}\right. \\
\times\left(\left\{\sum_{\tau=-\infty}^{n}[p(\tau)]^{-1 /(r-1)}\right\}^{r-1}\right. \\
\left.\left.+\left\{\sum_{\tau=n+1}^{+\infty}[p(\tau)]^{-1 /(r-1)}\right\}^{r-1}\right)^{-1}\right) q^{+}(n) \geq 1
\end{aligned}
$$

Corollary 5. Suppose that $r>1$ and $p(n)>0$ for $n \in \mathbb{Z}$, and that (44) holds. If (8) has a solution $x(n)$ satisfying (45), then

$$
\sum_{n=-\infty}^{+\infty} q^{+}(n)\left\{\sum_{\tau=-\infty}^{n}[p(\tau)]^{-1 /(r-1)} \sum_{\tau=n+1}^{+\infty}[p(\tau)]^{-1 /(r-1)}\right\}^{(r-1) / 2} \geq 2 .
$$

\section{Lyapunov-Type Inequalities for System (5)}

When $\mu=v=2$, assumption (B1) reduces the following form:

$$
\text { (B2) } \begin{aligned}
& \sum_{\tau=-\infty}^{0} \beta(\tau) \prod_{s=\tau}^{0}[1-\alpha(s)]^{-2} \\
& +\sum_{\tau=1}^{+\infty} \beta(\tau) \prod_{s=0}^{\tau-1}[1-\alpha(s)]^{2}<+\infty .
\end{aligned}
$$

Applying the results obtained in last section to the firstorder linear Hamiltonian system (5), we have immediately the following corollaries. 
Corollary 6. Suppose that hypotheses (A0), (B0), and (B2) are satisfied. If system (5) has a solution $(x(n), y(n))$ satisfying

$$
0<\sum_{n=-\infty}^{+\infty}\left[|x(n)|^{2}+(1+\beta(n))|y(n)|^{2}\right]<+\infty,
$$

then

$$
\begin{aligned}
\sum_{n=-\infty}^{+\infty}\left(\left\{\sum_{\tau=-\infty}^{n} \beta(\tau) \prod_{s=\tau}^{n}[1-\alpha(s)]^{-2}\right\}\right. \\
\times\left\{\sum_{\tau=n+1}^{+\infty} \beta(\tau) \prod_{s=n+1}^{\tau-1}[1-\alpha(s)]^{2}\right\} \\
\times\left(\sum_{\tau=-\infty}^{n} \beta(\tau) \prod_{s=\tau}^{n}[1-\alpha(s)]^{-2}\right. \\
\left.\left.\quad+\sum_{\tau=n+1}^{+\infty} \beta(\tau) \prod_{s=n+1}^{\tau-1}[1-\alpha(s)]^{2}\right)^{-1}\right) \gamma^{+}(n) \geq 1 .
\end{aligned}
$$

Corollary 7. Suppose that hypotheses (A1), (B0), and (B2) are satisfied. If system (5) has a soldution $(x(n), y(n))$ satisfying (48), then

$$
\sum_{n=-\infty}^{+\infty} \gamma^{+}(n)\left[\sum_{\tau=-\infty}^{n} \beta(\tau) \sum_{\tau=n+1}^{+\infty} \beta(\tau)\right]^{1 / 2} \geq 2 \prod_{n=-\infty}^{+\infty} \Theta[\alpha(n)] .
$$

Corollary 8. Suppose that $p(n)>0$ for $n \in \mathbb{Z}$, and that

$$
\sum_{\tau=-\infty}^{+\infty} \frac{1}{p(\tau)}<+\infty
$$

If (7) has a solution $x(n)$ satisfying

$$
0<\sum_{n=-\infty}^{+\infty}\left[|x(n)|^{2}+p(n)(1+p(n))|\Delta x(n)|^{2}\right]<+\infty
$$

then

$$
\sum_{n=-\infty}^{+\infty} q^{+}(n)\left[\sum_{\tau=-\infty}^{n} \frac{1}{p(\tau)} \sum_{\tau=n+1}^{+\infty} \frac{1}{p(\tau)}\right] \geq \sum_{n=-\infty}^{+\infty} \frac{1}{p(n)} .
$$

\section{Nonexistence of Homoclinic Solutions}

Applying the results obtained in Sections 2 and 3, we can drive the following criteria for non-existence of homoclinic solutions of systems (5) and (6) immediately.

Corollary 9. Suppose that hypotheses (AO), (B0), and (B1) are satisfied. If

$$
\sum_{n=-\infty}^{+\infty} \frac{\zeta(n) \eta(n)}{\zeta(n)+\eta(n)} \gamma^{+}(n)<1
$$

then system (6) has no solution $(x(n), y(n))$ satisfying

$$
0<\sum_{n=-\infty}^{+\infty}\left[|x(n)|^{\nu}+(1+\beta(n))|y(n)|^{\mu}\right]<+\infty
$$

Corollary 10. Suppose that hypotheses (A1), (B0), and (B1) are satisfied. If

$$
\sum_{n=-\infty}^{+\infty} \gamma^{+}(n)\left[\sum_{\tau=-\infty}^{n} \beta(\tau) \sum_{\tau=n+1}^{+\infty} \beta(\tau)\right]^{\nu / 2 \mu}<2 \prod_{n=-\infty}^{+\infty}\{\Theta[\alpha(n)]\}^{\nu / 2}
$$

then system (6) has no solution $(x(n), y(n))$ satisfying (55).

Corollary 11. Suppose that hypotheses (A1), (B0), and (B1) are satisfied. If

$$
\left(\sum_{n=-\infty}^{+\infty} \beta(n)\right)^{1 / \mu}\left(\sum_{n=-\infty}^{+\infty} \gamma^{+}(n)\right)^{1 / \nu}<2 \prod_{n=-\infty}^{+\infty}\{\Theta[\alpha(n)]\}^{1 / 2},
$$

then system (6) has no solution $(x(n), y(n))$ satisfying (55).

Corollary 12. Suppose that hypotheses (AO), (B0), and (B2) are satisfied. If

$$
\begin{aligned}
\sum_{n=-\infty}^{+\infty}\left(\left\{\sum_{\tau=-\infty}^{n} \beta(\tau) \prod_{s=\tau}^{n}[1-\alpha(s)]^{-2}\right\}\right. \\
\times\left\{\sum_{\tau=n+1}^{+\infty} \beta(\tau) \prod_{s=n+1}^{\tau-1}[1-\alpha(s)]^{2}\right\} \\
\times\left(\sum_{\tau=-\infty}^{n} \beta(\tau) \prod_{s=\tau}^{n}[1-\alpha(s)]^{-2}\right. \\
\left.\left.+\sum_{\tau=n+1}^{+\infty} \beta(\tau) \prod_{s=n+1}^{\tau-1}[1-\alpha(s)]^{2}\right)^{-1}\right) \gamma^{+}(n)<1,
\end{aligned}
$$

then system (5) has no solution $(x(n), y(n))$ satisfying

$$
0<\sum_{n=-\infty}^{+\infty}\left[|x(n)|^{2}+(1+\beta(n))|y(n)|^{2}\right]<+\infty
$$

Corollary 13. Suppose that hypotheses (A1), (B0), and (B2) are satisfied. If

$$
\sum_{n=-\infty}^{+\infty} \gamma^{+}(n)\left[\sum_{\tau=-\infty}^{n} \beta(\tau) \sum_{\tau=n+1}^{+\infty} \beta(\tau)\right]^{1 / 2}<2 \prod_{n=-\infty}^{+\infty} \Theta[\alpha(n)]
$$

then system (5) has no solution $(x(n), y(n))$ satisfying (59). 
Corollary 14. Suppose that $p(n)>0$ for $n \in \mathbb{Z}$, and that (51) holds. If

$$
\sum_{n=-\infty}^{+\infty} q^{+}(n)\left(\sum_{\tau=-\infty}^{n} \frac{1}{p(\tau)} \sum_{\tau=n+1}^{+\infty} \frac{1}{p(\tau)}\right)<\sum_{n=-\infty}^{+\infty} \frac{1}{p(n)},
$$

then (7) has no solution $x(n)$ satisfying (52).

Example 15. Consider the second-order difference equation:

$$
\Delta\left[\left(1+n^{2}\right) \Delta x(n)\right]+q(n) x(n+1)=0,
$$

where $q(n)$ is real-valued function defined on $\mathbb{Z}$. In view of Corollary 14, if

$$
\sum_{n=-\infty}^{+\infty}\left[\left(\sum_{\tau=-\infty}^{n} \frac{1}{1+\tau^{2}} \sum_{\tau=n+1}^{+\infty} \frac{1}{1+\tau^{2}}\right)\right] q^{+}(n)<\sum_{n=-\infty}^{+\infty} \frac{1}{1+n^{2}},
$$

then (62) has no solution $x(n)$ satisfying

$$
0<\sum_{n=-\infty}^{+\infty}\left[|x(n)|^{2}+\left(1+n^{2}\right)^{2}|\Delta x(n)|^{2}\right]<+\infty .
$$

\section{Acknowledgment}

This work is supported by the Scientific Research Fund of Hunan Provincial Education Department (07A066).

\section{References}

[1] A. M. Lyapunov, "Probléme général de la stabilité du mouvement," Annde La Faculté, vol. 2, no. 9, pp. 203-474, 1907.

[2] M. Bohner, S. Clark, and J. Ridenhour, "Lyapunov inequalities for time scales," Journal of Inequalities and Applications, vol. 7, no. 1, pp. 61-77, 2002.

[3] S. S. Cheng, "A discrete analogue of the inequality of Lyapunov," Hokkaido Mathematical Journal, vol. 12, no. 1, pp. 105-112, 1983.

[4] S.-S. Cheng, "Lyapunov inequalities for differential and difference equations," Polytechnica Posnaniensis, no. 23, pp. 25-41, 1991.

[5] S. Clark and D. Hinton, "A Liapunov inequality for linear Hamiltonian systems," Mathematical Inequalities \& Applications, vol. 1, no. 2, pp. 201-209, 1998.

[6] S. Clark and D. Hinton, "Discrete Lyapunov inequalities," Dynamic Systems and Applications, vol. 8, no. 3-4, pp. 369-380, 1999.

[7] G. Sh. Guseinov and B. Kaymakçalan, "Lyapunov inequalities for discrete linear Hamiltonian systems," Computers \& Mathematics with Applications, vol. 45, no. 6-9, pp. 1399-1416, 2003.

[8] G. Sh. Guseinov and A. Zafer, "Stability criteria for linear periodic impulsive Hamiltonian systems," Journal of Mathematical Analysis and Applications, vol. 335, no. 2, pp. 1195-1206, 2007.

[9] P. Hartman, "Difference equations: disconjugacy, principal solutions, Green's functions, complete monotonicity," Transactions of the American Mathematical Society, vol. 246, pp. 1-30, 1978.

[10] X. He and Q.-M. Zhang, "A discrete analogue of Lyapunovtype inequalities for nonlinear difference systems," Computers \& Mathematics with Applications, vol. 62, no. 2, pp. 677-684, 2011.
[11] L. Jiang and Z. Zhou, "Lyapunov inequality for linear Hamiltonian systems on time scales," Journal of Mathematical Analysis and Applications, vol. 310, no. 2, pp. 579-593, 2005.

[12] S. H. Lin and G. S. Yang, "On discrete analogue of Lyapunov inequality," Tamkang Journal of Mathematics, vol. 20, no. 2, pp. 169-186, 1989.

[13] X. Wang, "Stability criteria for linear periodic Hamiltonian systems," Journal of Mathematical Analysis and Applications, vol. 367, no. 1, pp. 329-336, 2010.

[14] X.-H. Tang and M. Zhang, "Lyapunov inequalities and stability for linear Hamiltonian systems," Journal of Differential Equations, vol. 252, no. 1, pp. 358-381, 2012.

[15] X. H. Tang, Q.-M. Zhang, and M. Zhang, "Lyapunov-type inequalities for the first-order nonlinear Hamiltonian systems," Computers \& Mathematics with Applications, vol. 62, no. 9, pp. 3603-3613, 2011.

[16] A. Tiryaki, M. Ünal, and D. Çakmak, "Lyapunov-type inequalities for nonlinear systems," Journal of Mathematical Analysis and Applications, vol. 332, no. 1, pp. 497-511, 2007.

[17] M. Ünal, D. Çakmak, and A. Tiryaki, "A discrete analogue of Lyapunov-type inequalities for nonlinear systems," Computers \& Mathematics with Applications, vol. 55, no. 11, pp. 2631-2642, 2008.

[18] M. Ünal and D. Çakmak, "Lyapunov-type inequalities for certain nonlinear systems on time scales," Turkish Journal of Mathematics, vol. 32, no. 3, pp. 255-275, 2008.

[19] Q.-M. Zhang and X. H. Tang, "Lyapunov inequalities and stability for discrete linear Hamiltonian systems," Applied Mathematics and Computation, vol. 218, no. 2, pp. 574-582, 2011.

[20] Q.-M. Zhang and X. H. Tang, "Lyapunov inequalities and stability for discrete linear Hamiltonian systems," Journal of Difference Equations and Applications, vol. 18, no. 9, pp. 14671484, 2012.

[21] R. Agarwal, C. Ahlbrandt, M. Bohner, and A. Peterson, "Discrete linear Hamiltonian systems: a survey," Dynamic Systems and Applications, vol. 8, no. 3-4, pp. 307-333, 1999.

[22] C. D. Ahlbrandt and A. C. Peterson, Discrete Hamiltonian Systems, vol. 16 of Kluwer Texts in the Mathematical Sciences, Kluwer Academic, Dordrecht, The Netherlands, 1996.

[23] S. N. Elaydi, An Introduction to Difference Equations, Springer, New York, NY, USA, 3rd edition, 2004. 


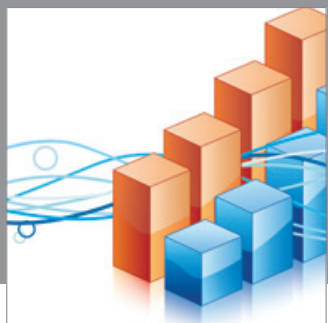

Advances in

Operations Research

mansans

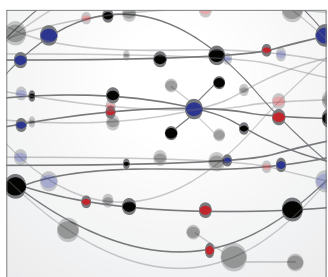

The Scientific World Journal
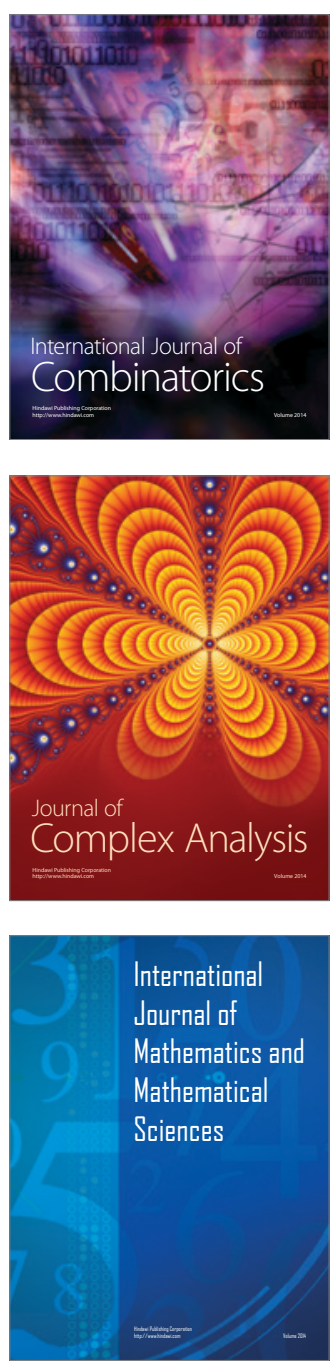
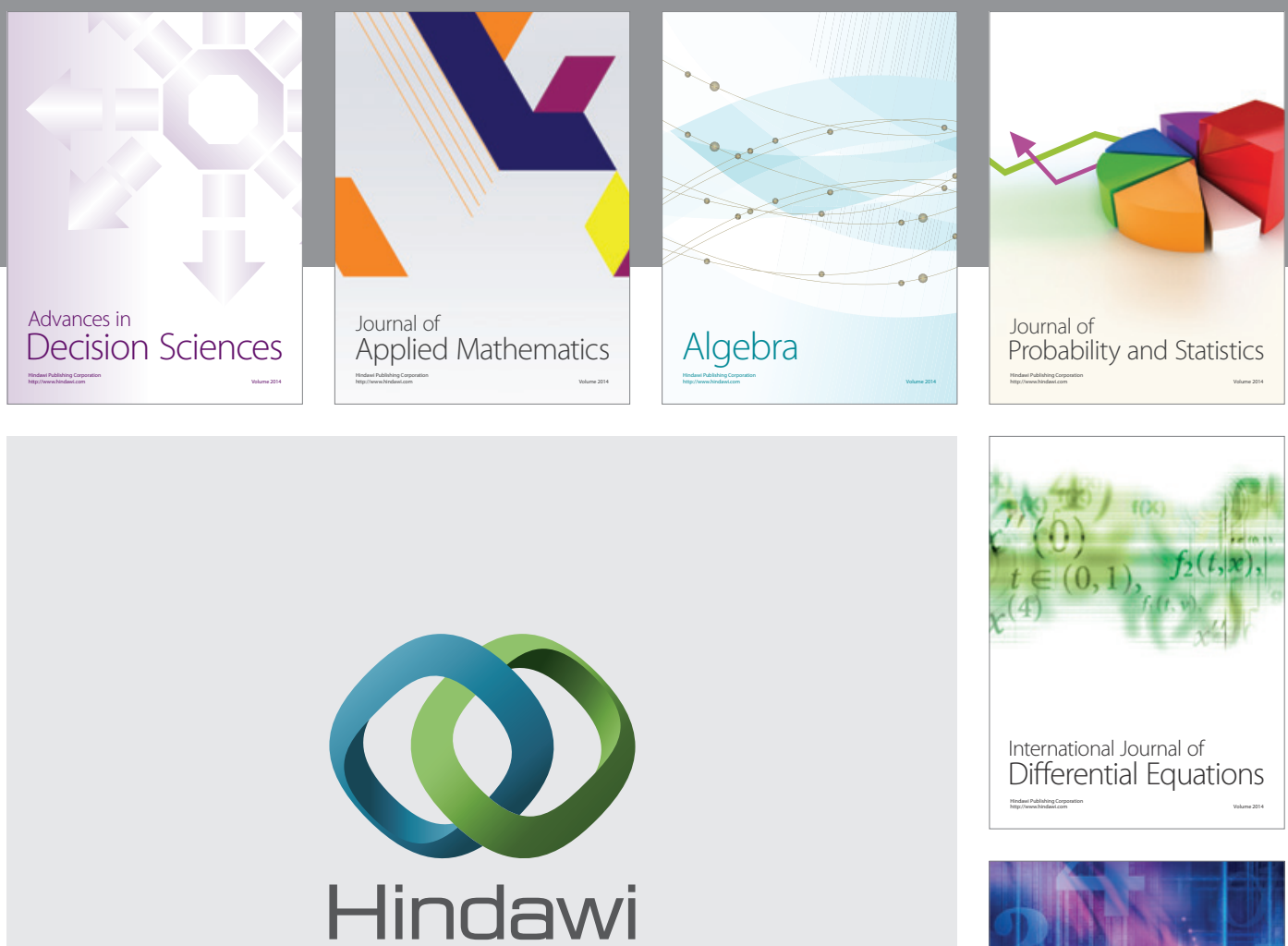

Submit your manuscripts at http://www.hindawi.com
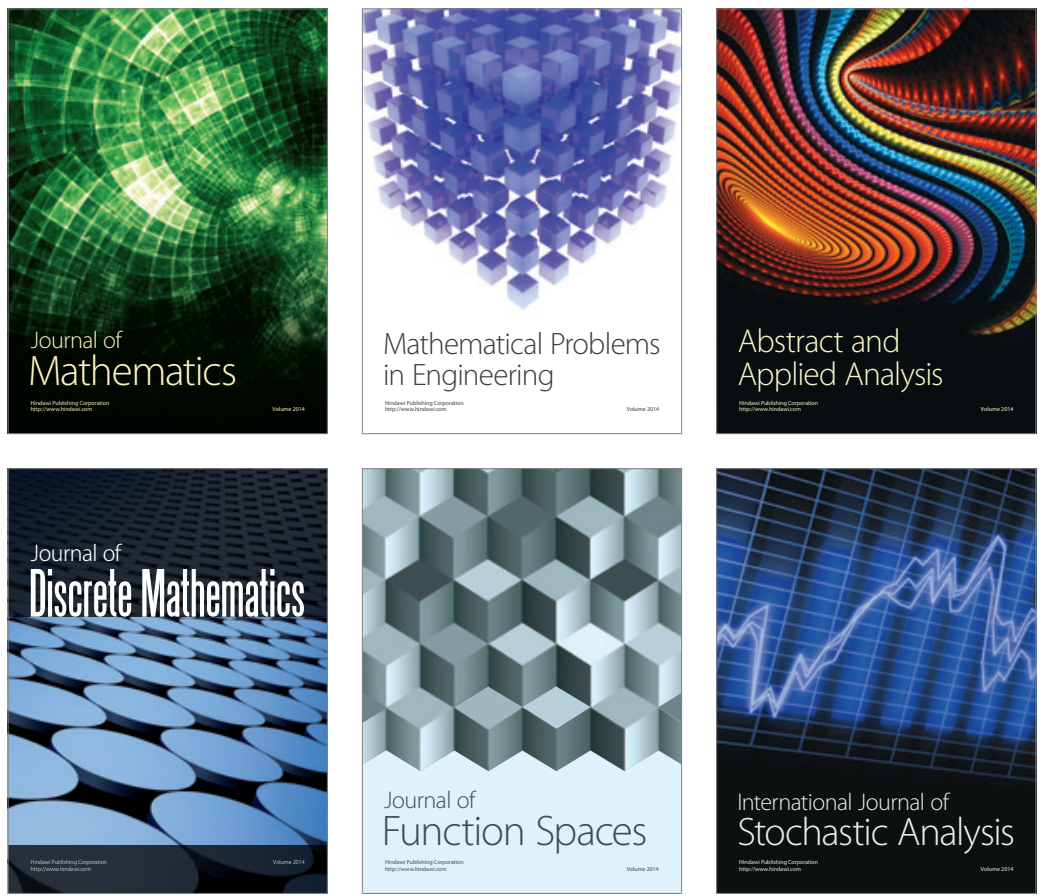

Journal of

Function Spaces

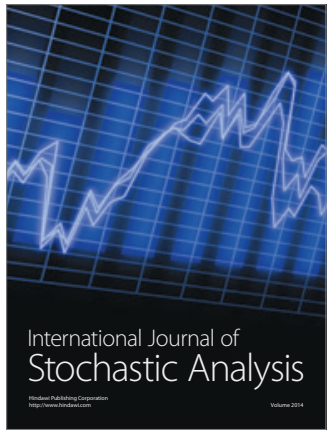

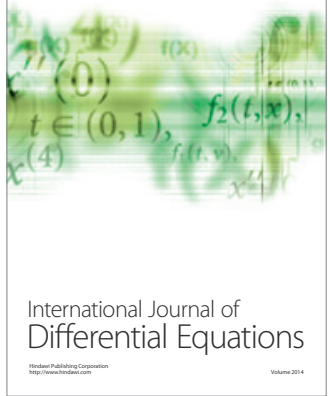
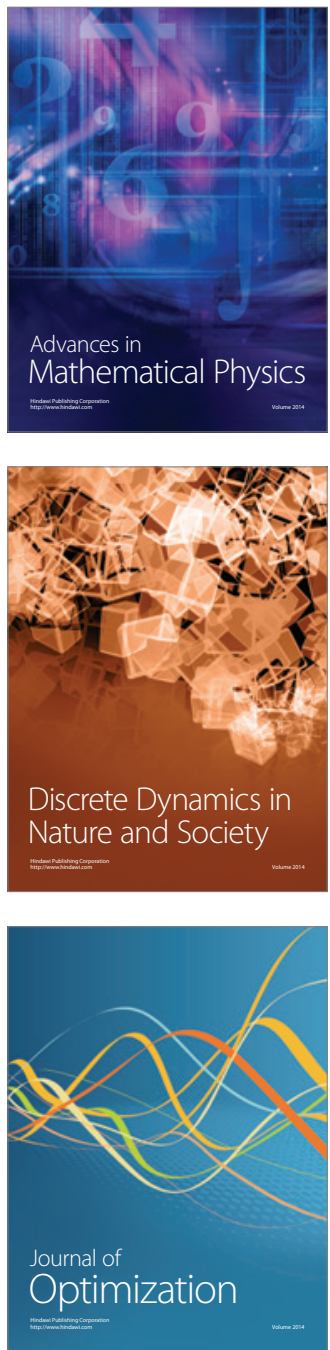